\title{
Fabrication of a High-flux Sulfonated Polyamide Nanofiltration Membrane: Experimental and Dissipative Particle Dynamics Studies
}

\author{
Jiahui Hu, Zhiwei Lv, Yunzhi Xu, Xuan Zhang* and Lianjun Wang* \\ Jiangsu Key Laboratory of Chemical Pollution Control and Resources \\ Reuse, School of Environmental and Biological Engineering, Nanjing \\ University of Science \& Technology, Nanjing 210094, China.
}

\begin{abstract}
Novel high-flux thin film composite (TFC) nanofiltration (NF) membranes were fabricated via interfacial polymerization (IP) on a polysulfone substrate using 2,2'-benzidinedisulfonic acid (BDSA) as the amine monomer in aqueous solution. The intrinsic resistance of the resulting membranes $\left(R_{\mathrm{m}}\right)$ was found to be as low as $2.37 \times 10^{13} \mathrm{~m}^{-1}$, indicating a considerably low tolerance against hydraulic resistance. The optimized membrane TFC-1.0 showed excellent rejection towards different inorganic electrolyte solutions in the following order: $\mathrm{Na}_{2} \mathrm{SO}_{4}>\mathrm{NaCl}>\mathrm{MgCl}_{2}>$ $\mathrm{CuSO}_{4}>\mathrm{MgSO}_{4}$, indicating the negatively charged nature of the membrane. Surface properties were evaluated by field emission scanning electron microscopy (FE-SEM), atomic force microscopy (AFM) and contact angle measurements, and the results indicated a smooth and moderately hydrophobic surface. Using dissipative particle dynamics (DPD), the coarse-grained model molecules at the mesoscale level were used to establish an oil-water interface model with regard to the current IP process. The orientation of BDSA monomers was studied and it was found that most of the sulfonic acid groups were facing towards the aqueous solution, while the outer faces were covered by the nanoaggregates of the rigid polymer backbones. This finding is
\end{abstract}


consistent with the observed experimental results and provides a reasonable explanation for the surface properties. The NF membrane has a high water permeability of $16.6 \mathrm{~L} \mathrm{~m}^{-2} \mathrm{~h}^{-1} \mathrm{bar}^{-1}$ and high rejection ability for inorganic salts and organic solutes. These results confirm the suitability of this membrane for various applications, such as desalination and dye removal from wastewater.

\section{Keywords:}

Sulfonated Polyamide; Nanofiltration; High performance; Thin film composite; Dissipative particle dynamics 


\section{Introduction}

Nanofiltration (NF) is a relatively new pressure-driven membrane separation technology with efficiency in between that of ultrafiltration and reverse osmosis. NF was first introduced during the late 1980s, and generally exhibits the favorable separation characteristics of high permeation flux and retention of multivalent salts and organics with molecular weight greater than $200 \mathrm{Da}$ [1-8]. Due to its low pressure operation, mild working conditions and adaptability to different applications, the NF process has been gradually adopted in several research areas including water softening, organic pollutants recovery and heavy metal removal [9-13].

Most of the present day commercially available NF membranes are derived from cross-linked aromatic polyamides (PA) through interfacial polymerization (IP) between acyl chlorides and various water-soluble reactants [14-17]. The key merit for this process is that the individual characteristics of the active thin layer could be separately optimized to achieve a better separation performance. It is generally considered that the polymeric structure at the surface directly determines the separation performance in terms of water permeability and solute rejection [18-22]. Therefore, the IP process has been extensively studied in recent years, particularly in evaluating new functional precursors.

For instance, Jiang et al. prepared TFC membranes using diethylenetriamine, triethylenetetramine, tetraethylenepentamine and piperazidine as the small molecular monomers. Their results showed that the higher the solubility of the amine-monomers in the organic solvent, the greater would be the pore density and permeability of the resulting membrane [23]. Wang and his team also investigated a polyelectrolyte, i.e., branched polyethyleneimine as the starting material. The resulting membranes were positively charged when placed in neutral water, and thus rather strongly rejected $\mathrm{Mg}^{2+}$ and $\mathrm{Ca}^{2+}$ ions [24].

However, to date, most of the NF related studies are focused on the separation performance of the membrane, i.e., the permeate flux and rejection properties. Due to the increasing usage of membrane technology in water treatment processes, it is 
important to improve our understanding of synthetic membranes and separation mechanisms. One convenient method to achieve this objective is to employ computational techniques [25-30]. For instance, Lo et al. used a quantum mechanical simulation to calculate the global/local reactivity of piperazine (PIP) and m-phenylenediamine (MPD) molecules towards trimesoyl chloride (TMC) based on density functional theory (DFT). It was found that the reaction between MPD and TMC for the preparation of TFC membrane was more favorable than the reaction between PIP and TMC. The reason for this is possibly the greater number of unreacted carboxylic groups left, resulting in a more charged surface, in turn leading to better selectivity of bivalent salt rejection for the latter [27]. More recently, the properties of a hydrated amorphous PA membrane-foulant system were investigated using molecular dynamics (MD) simulations by Leng et al. [28]. The authors found that the carboxylate groups in both the PA surface and alginate exhibit strong binding with metal ions, and the predominantly existing bidentate configuration leads to the surface fouling. A more interesting method has been reported by Roux's group, in which only TMC and MPD monomers were initially mixed in a box, followed by a polymerization process based on heuristic distance criterion obtained from MD simulations [29]. The atomic density in the model membrane was found to be close to the reference value and the water permeability agreed well with the experiment data, suggesting the reliability of their method. In general, while there are increasing number of reports on the simulations, most of these studies focus on the method improvements or describing the structures based on some commercially available membranes. There is limited information regarding the newly-designed membranes. Furthermore, whether the simulation is performed on the atomic or molecular level, the fundamental aim is to reveal the interactions between the functional groups, particles or water molecules, in order to understand the molecular movement on and through the membranes. In other words, these simulation methods are intended to provide us with a deeper understanding of the phenomena in membrane-based water treatment processes. However, the major drawback is that simulation is a time consuming process, especially when the system contains ten thousand atoms or more. 
Due to the inherent length and time scale of the simulation process, it would be difficult to monitor the molecular diffusion and self-assembly behaviors during the membrane forming process.

Clearly, there is a definite need to obtain an overall picture of the important aspects such as the structure-property relationship of the monomer, or the polymeric precursors with regard to the IP process. For example, what would be the real geometry of the precursors, what kind of diffusion behavior would they exhibit, and what might be the orientation of the precursors once they came into contact and reacted with the aryl chloride in the organic phase? Only after having understood the answers to these questions, would it be possible to obtain clear structural configurations.

In this work, we used BDSA as a typical water-soluble monomer in the IP process for fabricating the TFC membranes. The unique structure of BDSA based on a hydrophobic phenyl-phenyl backbone and two hydrophilic sulfonic acid groups, could effectively help us explore the diffusion process as well as the final properties of the active sulfonated polyamide (SPA) layer. The TFC membranes were prepared via the IP process, for the purpose of desalination. The separation properties of these membranes were then evaluated by determination of the permeate flux and rejection ability for salts and dye solutions. Moreover, the dissipative particle dynamics (DPD) simulation was performed by adopting the concept of an oil-water two-phase interface. The complex interactions among the monomers, organic solutions and aqueous solutions were neglected and replaced by the repulsion between the motion groups. In this way, the diffusion process of the BDSA molecules and their orientation in the SPA layer were clearly illustrated. Finally, through a combination of the separation properties and DPD results, a new configuration model was proposed in regards to our BDSA/TMC NF membrane system.

\section{Experimental}

\subsection{Materials}


BDSA, Sodium laurylsulfonate (SDS), Triethylamine (TEA), Congo red (MW: 696.68 Da), Methylene blue (MW: 319.86 Da) and Bovine serum albumin (BSA) were purchased from Sinoparm Chemical Reagent Co. Ltd. (China). TMC, ethylene glycol, diethylene glycol, triethylene glycol, and glucose were purchased from J\&K Chemical Reagent Co. Ltd. (Beijing, China). Polysulfone (PSf) ultrafiltration membranes (Molecular weight cut-off: 20000; Water flux: $120 \mathrm{~L} \mathrm{~m}^{-2} \mathrm{~h}^{-1}, 0.1 \mathrm{MPa}$ ) were supplied from Development Center of Water Treatment Technology (Hangzhou, China) and used as a support membrane. Sodium sulfate, Magnesium sulfate, Magnesium chloride hexahydrate, Sodium chloride, Copper(II) sulfate pentahydrate and $n$-hexane were purchased from Nanjing Chemical Reagent Co. Ltd. (Nanjing, China). All other chemicals were obtained from commericial sourses and used without further purification. Deionized water was used throughout this study.

\subsection{Procedure of membrane preparation}

The active skin layer of TFC membrane was prepared by conventional interfacial polymerization technology at room temperature about $25^{\circ} \mathrm{C}$. A PSf support membrane was immersed in deionized water overnight, then removed from the water and fixed on a plastic plate. First, the aqueous-phase solution containing a certain amount of BDSA and $0.04 \%(w / w)$ of SDS was prepared with $\mathrm{pH} 10$ adjusted by TEA. The organic-phase solution was prepared with a certain amount of TMC in $n$-hexane at room temperature. $100 \mathrm{~mL}$ of BDSA solution were poured into the frame and allowed to contact the PSf membrane for 5 min before draining the excess aqueous solution. This residence time allowed BDSA to penetrate into the pores of the porous support. Residual droplets of solution on the top surface of the PSf membrane were removed by rolling a rubber roller across the membrane surface. Afterwards, the frame and gasket were reassembled on top of the PSf membrane, and $100 \mathrm{~mL}$ of TMC solution were poured into the frame. After $1 \mathrm{~min}$, the TMC solution was drained off and the membrane surface was rinsed using $n$-hexane $(100 \mathrm{~mL})$ to wash away residual reagents. Finally the membrane was dried in air at ambient conditions for $30 \mathrm{~s}$, immersed in a weak acidity aqueous solution for several hours, and saved in deionized water before carrying out for evaluation studies. 


\subsection{Membrane characterization}

Surface chemical characterization was performed using X-ray photoelectron spectroscope (XPS, PHI Quantera II, Japan). Square-shaped coupons with approximate dimensions $1 \mathrm{~cm} \times 1 \mathrm{~cm}$ were cut from each of the coated membranes and mounted on the XPS stage. The survey scan was performed in the binding energy range $0-1000 \mathrm{eV}$ with a resolution of $1 \mathrm{eV}$. High resolution scans of $\mathrm{C}(1 \mathrm{~s}), \mathrm{N}(1 \mathrm{~s}), \mathrm{O}$ (1s) and S (2p) were conducted under similar conditions with $0.05 \mathrm{eV}$ steps, pass energy $20 \mathrm{eV}$.

Surface morphology and cross-sections were examined using Field Emission Scanning Electron Microscopy (FE-SEM, FEI Quanta 250F, America). The samples for SEM were coated with a thin layer of gold to make them conducting.

Surface roughness was determined quantitatively using an Atomic Force Microscope (AFM, Mutilmode8, German) equipped with a standard SiN cantilever. Dry membrane samples of dimensions $1 \mathrm{~cm} \times 1 \mathrm{~cm}$ were mounted on a glass plate using double-sided tape. For better accuracy and precision, measurements were performed at different locations and for variable scan areas. Surface roughness was reported in terms of the average plane roughness $(\mathrm{Ra})$, root-mean-square (RMS) and the relative surface area.

Contact angle measurements were performed using a Contact Angle Measurement Device (KRÜSS DSA30, German) with FAMAS Interface Measurement \& Analysis System version 3.1.3. The sessile drop method was used to measure the contact angle of a $20 \mu \mathrm{L}$ water droplet placed carefully on the flat membrane surface. A total of 8 measurements at different locations were carried out for each sample.

A streaming potential method was utilized to detect the charging property of the membrane surface using the electrokinetic analyzer (SurPASS Anton Paar, Austria) with $\mathrm{KCl}\left(1 \mathrm{mmol} \mathrm{L}^{-1}\right)$ solution as electrolyte solution. The $\mathrm{pH}$ dependence of surface zeta potential was investigated via adjusting $\mathrm{pH}$ by $\mathrm{NaOH}$ and $\mathrm{HCl}$ solutions. The surface zeta potential $\zeta$ was determined by the Helmholtz-Smoluchowski equation:

$$
\zeta=\frac{\Delta E}{\Delta P} \frac{\eta \kappa}{\varepsilon}
$$


where $\Delta E$ is streaming potential, $\Delta P$ is pressure, $\varepsilon$ is the dielectric constant, $\eta$ and $\kappa$ are the viscosity and the conductivity of the solution, respectively.

\subsection{Membrane separation performance tests}

The experiments of TFC membranes to treatment of pure water or salt solution were performed at 6 bar using pure water or $1000 \mathrm{mg} \mathrm{L}^{-1}$ salt or organic solution by self-made cross-flow equipment at room temperature. The effective membrane area is around $12.56 \mathrm{~cm}^{2}$. The TFC membranes were initially subjected to pure water with pressure of 10 bar for $1 \mathrm{~h}$ prior to performing the TFC performance testing experiments. The pure water or salt solution flux, $J\left(\mathrm{~L} \mathrm{~m}^{-2} \mathrm{~h}^{-1}\right)$ of the membrane was determined by direct measurement of permeate volume, which was calculated by the following equation:

$$
J=\frac{V}{A \Delta t}
$$

where $V$ was the volume of permeated water, $A$ was the effective membrane area and $\Delta t$ was the permeation time.

Ethylene glycol, diethylene glycol, triethylene glycol and glucose were used to measure the pore size and pore size distribution of the TFC membrane. Solutions containing $1000 \mathrm{mg} \mathrm{L}^{-1}$ of these organic solutes were prepared and used as feed solutions. During tests, each feed solution was allowed to stabilize for $1 \mathrm{~h}$ at $10 \mathrm{bar}$ before sample collection and was analyzed by a total organic carbon analyzer (vario TOC cube, German). The pore size and its distribution, the mean effective pore radius $\left(\mu_{\mathrm{p}}\right)$ and the geometric standard deviation $\left(\sigma_{\mathrm{p}}\right)$ of the membrane were therefore determined by the correlation between rejection and radius of the four solutes as described elsewhere $[10,31-33] . \mu_{\mathrm{p}}$ is equal to the radius of the solute at $\mathrm{R}=50 \%$, and $\sigma_{\mathrm{p}}$ is the ratio of the solute radius when $\mathrm{R}=84.13 \%$ over the solute radius when $\mathrm{R}=50 \%$. Then the pore size distribution of the membrane can be expressed as follows:

$$
\frac{d R_{T}\left(r_{p}\right)}{d r_{p}}=\frac{1}{r_{p} \ln \sigma_{p} \sqrt{2 \pi}} \exp \left[-\frac{\left(\ln r_{p}-\ln \mu_{p}\right)^{2}}{2\left(\ln \sigma_{p}\right)^{2}}\right]
$$

where $r_{\mathrm{p}}$ is the effective pore radius of the membrane. The values of $\mu_{\mathrm{p}}$ and $\sigma_{\mathrm{p}}$ determine the position and sharpness of the distribution curves, respectively. 
The salt solution concentration of permeation was measured by Conductivity Meter DDS-307 (Shanghai, China). The dye solution concentration of permeation was measured by UV/VIS Spectrometer (Lambda 25, PerkinElmer). $\mathrm{CuSO}_{4}$ solution was measured by atomic absorption spectrometry. The membrane rejection $(R)$ was calculated by using the following equation:

$$
R=\left(1-\frac{C_{p}}{C_{f}}\right) \times 100 \%
$$

In which $C_{\mathrm{p}}\left(\mathrm{mg} \mathrm{L}^{-1}\right)$ is the permeate concentration and $C_{\mathrm{f}}\left(\mathrm{mg} \mathrm{L}^{-1}\right)$ is the feed concentration. All membrane samples were prepared and tested in at least two measurements, results of which have been averaged.

The membrane hydraulic resistance $\left(R_{\mathrm{m}}\right)$ was determined using the relationship from equation (5):

$$
J=\frac{\Delta P}{\mu R_{m}}
$$

where $J$ is the pure water flux $\left(\mathrm{L} \mathrm{m}^{-2} \mathrm{~h}^{-1}\right), \Delta P$ is the transmembrane pressure (Pa) and $\mu$ is the dynamic viscosity of water ( $\mathrm{Pa} \mathrm{s}$ ). Membrane resistance was determined from a linear regression of the measured pure water flux and transmembrane pressure data.

To characterize the antifouling properties of membranes, BSA aqueous solution (100 mg L $\mathrm{L}^{-1}$ ) was used. The long-time antifouling experiment mainly included three steps: the pure water flux $J_{\mathrm{w} 1}\left(\mathrm{~L} \mathrm{~m}^{-2} \mathrm{~h}^{-1}\right)$ was first measured at a transmembrane pressure of 0.6 MPa for $30 \mathrm{~min}$. Then, the flux for BSA solution $J_{\mathrm{P}}\left(\mathrm{L} \mathrm{m}^{-2} \mathrm{~h}^{-1}\right)$ was measured under 0.6 MPa for $30 \mathrm{~min}$. In third step, the polluted membranes were washed for $1 \mathrm{~h}$ with deionized water, and the pure water flux of cleaned membranes $J_{\mathrm{w} 2}\left(\mathrm{~L} \mathrm{~m}^{-2} \mathrm{~h}^{-1}\right)$ for $1 \mathrm{~h}$ was measured.

The flux recovery ratio (FRR) and total flux decline ratio $\left(D R_{\mathrm{t}}\right)$ were used to evaluate the antifouling property of the membrane. They were defined as follows:

$$
F R R=\left(\frac{J_{W 2}}{J_{W 1}}\right) \times 100 \%
$$




$$
D R_{t}=\left(\frac{J_{W 1}-J_{P}}{J_{W 1}}\right) \times 100 \%
$$

\subsection{DPD simulations}

Four different components were built in our system: water, $n$-hexane, TMC and BDSA. Calculations of repulsion parameters were made using molecular structures listed in Table 1. The coarse-graining level was defined and the DPD parameters were set according to the method published previously [34].

Table 1 Coarse-grained structure for the involved components.

\begin{tabular}{|c|c|c|c|c|c|}
\hline Solvent & Molecules & $\begin{array}{l}\text { Bead } \\
\text { type }\end{array}$ & $\begin{array}{l}\text { Molecular } \\
\text { structure }\end{array}$ & $\begin{array}{c}\text { Coarse-grained } \\
\text { structure }\end{array}$ & $\begin{array}{l}\text { Solubility } \\
\text { parameter } \\
\left(\mathrm{J} \mathrm{cm}^{-3}\right)^{1 / 2}\end{array}$ \\
\hline Water & $\mathrm{H}_{2} \mathrm{O}$ & $\mathrm{W}$ & & & $47.9^{\text {a) }}$ \\
\hline Aniline & $\mathrm{C}_{6} \mathrm{H}_{5} \mathrm{~N}$ & A & C & & $21.4^{\text {a) }}$ \\
\hline Methanesulfonic acid & $\mathrm{CH}_{3} \mathrm{SO}_{3} \mathrm{H}$ & $S$ & ○ & & $45.5^{\mathrm{b})}$ \\
\hline$n$-Hexane & $\mathrm{C}_{6} \mathrm{H}_{14}$ & $\mathrm{H}$ & & & $14.9^{\text {a) }}$ \\
\hline Benzene & $\mathrm{C}_{6} \mathrm{H}_{6}$ & B & C & & $18.6^{\mathrm{a})}$ \\
\hline Acetyl chloride & $\mathrm{CH}_{3} \mathrm{COCl}$ & $\mathrm{C}$ & ○ & & $19.4^{\text {a) }}$ \\
\hline
\end{tabular}

a) Obtained from ref [35];

b) Obtained from cohesive energy density (CED) result.

This work is focused on the study of the interfacial polymerization of the BDSA and TMC monomers, as described as an oil-water two phase interface. Therefore, a cubic simulation box with $100 \AA$ A side lengths is constructed. The simulation box with regard to the IP surface system is composed of two slabs (slabs 1 and 2, see Fig. S2) of average density of 3 in reduced units. In slab 1, we have placed a water phase layer $(100 \times 100 \times 50)$ composed of BDSA and water beads (BDSA/water $=0.2: 0.8)$ at the bottom of the simulation box. Above this latter slab, we have placed a layer (slab 2, 
$100 \times 100 \times 50)$ of TMC beads mixed with $n$-hexane bead $(\mathrm{TMC} / n$-hexane=0.05:0.95). Each region was independently relaxed before the system was assembled for simulations. The DPD calculations carried out in this work were performed using the Mesocite module in Materials Studio software.

\section{Results \& Discussion}

The reaction process of interfacial polymerization between BDSA and TMC was showed in Scheme 1. The nomenclature used for the series of TFC membranes is: TFC-x, where $\mathrm{x}$ refers to the concentration of BDSA in the aqueous solution, as listed in Table 2.

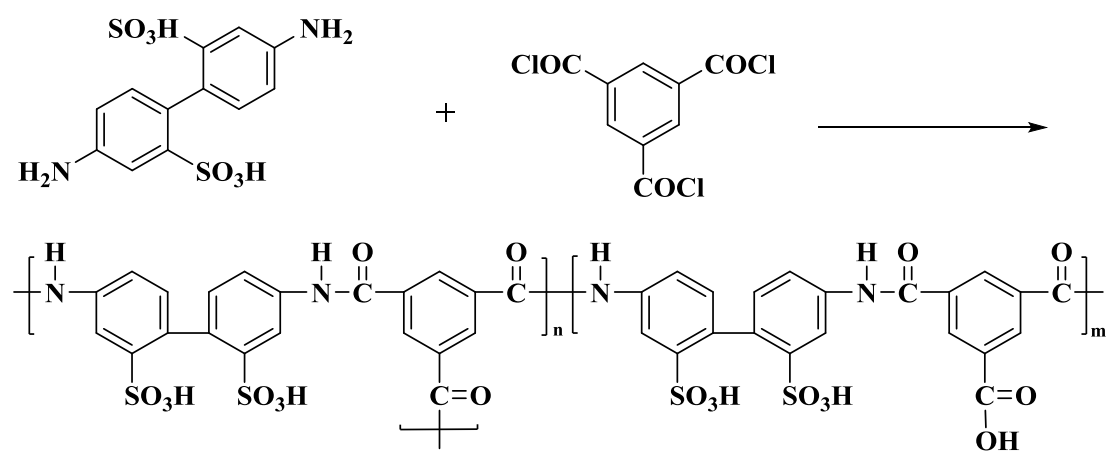

Scheme 1 The reaction process of interfacial polymerization between BDSA and TMC.

\subsection{Surface composition}

XPS analysis was used to characterize the chemical composition of the TFC surface, and the results are shown in Table 2. The $\mathrm{C}(1 \mathrm{~s}), \mathrm{N}(1 \mathrm{~s}), \mathrm{O}(1 \mathrm{~s})$, and $\mathrm{S}(2 \mathrm{p})$ atomic concentrations of the membranes were investigated. The peak occurring at $398.5 \mathrm{eV}$ in the $\mathrm{N} 1 \mathrm{~s}$ spectrum of TFC membranes can be attributed to the $\mathrm{C}-\mathrm{N}$ group in "-CO-NH-", indicating the amide bond formation. By calculating the $\mathrm{O} / \mathrm{N}$ ratio, the crosslinking state can be determined. The $\mathrm{O} / \mathrm{N}$ ratio in the present study gradually decreased from the upper limit of 5.0 as the BDSA feeding amount increased from 0.5 to $1.0 \%(\mathrm{w} / \mathrm{v})$, indicating the increase in degree of crosslinking in the active layer. It is known that high crosslinking extent of TFC membrane is required to reduce the solute 
permeability and achieve high rejections. Therefore, it can be concluded that the most cross-linked membrane TFC-1.0 should exhibit the best solute retention performance during the separation test.

Table 2 XPS result of PSf and TFC membranes.

\begin{tabular}{ccccccc}
\hline Code & $\mathrm{C}_{\mathrm{BDSA}}(\%)^{\mathrm{a})}$ & $\mathrm{C}(\%)$ & $\mathrm{O}(\%)$ & $\mathrm{N}(\%)$ & $\mathrm{S}(\%)$ & $\mathrm{O} / \mathrm{N}$ \\
\hline PSf & 0 & 81.1 & 16.0 & & 2.9 & \\
TFC-0.5 & 0.5 & 71.0 & 20.9 & 5.1 & 2.9 & 4.10 \\
TFC-0.7 & 0.7 & 71.7 & 17.9 & 6.4 & 2.9 & 2.80 \\
TFC-1.0 & 1.0 & 71.5 & 19.7 & 7.5 & 1.3 & 2.63 \\
TFC-1.2 & 1.2 & 69.9 & 20.4 & 5.7 & 4.0 & 3.58 \\
\hline
\end{tabular}

a) TMC concentration was fixed at $0.1 \%(\mathrm{w} / \mathrm{v})$.

\subsection{Membrane separation performance}

Water flux and salt rejection rate were measured to evaluate the basic performance of the TFC membranes. A series of TFC membranes were obtained by varying the BDSA and TMC concentrations, and their separation characteristics were extensively studied so as to optimize the experimental feed amount. Fig. 1 (a) shows the effects of BDSA concentration on the water flux and rejection properties of the resulting membrane at a fixed TMC concentration of $0.1 \%(\mathrm{w} / \mathrm{v})$. Initially, the model interfacial polymerization process was carried out with BDSA concentration starting from $0.1-0.4 \%(\mathrm{w} / \mathrm{v})$. However, this resulted in a loosely cross-linked polymer formed at the interface. The membrane formed with this material performed poorly and showed a huge water flux and negligible salt rejection. Therefore, the threshold concentration of amine monomer was chosen to be $0.5 \%(\mathrm{w} / \mathrm{v})$, and the resulting membrane showed a moderate rejection ability of $52.5 \%$ for $\mathrm{Na}_{2} \mathrm{SO}_{4}$. Upon increasing the concentration of BDSA from 0.5 to $1.0 \%(\mathrm{w} / \mathrm{v})$, the rejection of $\mathrm{Na}_{2} \mathrm{SO}_{4}$ also increased considerably, indicating the formation of the densest layer. Although the water flux decreased to the lowest value of $16.6 \mathrm{~L} \mathrm{~m}^{-2} \mathrm{~h}^{-1} \mathrm{bar}^{-1}$, this value is still toward the higher end of the range for NF membranes, particularly for flat sheet membrane series [36-38]. With further increase in the monomer concentration, the 
solubility of the resulting sulfonated polyamide becomes even lower in the organic phase due to the high polarity of "- $\mathrm{SO}_{3} \mathrm{H}$ " groups as well as the extremely high rigidity of the polymer chain. Therefore, the polymer precipitates within a short period of time with a large amount of the unreacted amine groups left, resulting in a low crosslinking density and a consequentially decreased salt rejection.

The effect of TMC concentration on the rejection and permeate flux properties of TFC membranes is shown in Fig. 1 (b), and it can be seen that the trends are similar to those of BDSA. At very low concentration of TMC $(0.05 \%(\mathrm{w} / \mathrm{v}))$, the membrane showed relatively low salt rejection of $80 \%$, which could be attributed to the low degree of crosslinking. When the TMC concentration was increased to $0.1 \%(\mathrm{w} / \mathrm{v})$, a thicker and more compact polyamide barrier layer was formed which was able to reject the inorganic salts more effectively. Compared to some other polyamide NF series been rich in functional groups (e.g., polyethyleneimine) [39, 40], the crosslinking density for our TFCs should be much lower, and thus exhibits rather high water permeability. Moreover, it is worth noting that the water permeation for $\mathrm{Na}_{2} \mathrm{SO}_{4}$ solution was slightly lower than that for pure water, as shown in Fig. 1 (a) and (b), which was attributed to its higher osmosis pressure higher than the latter. 

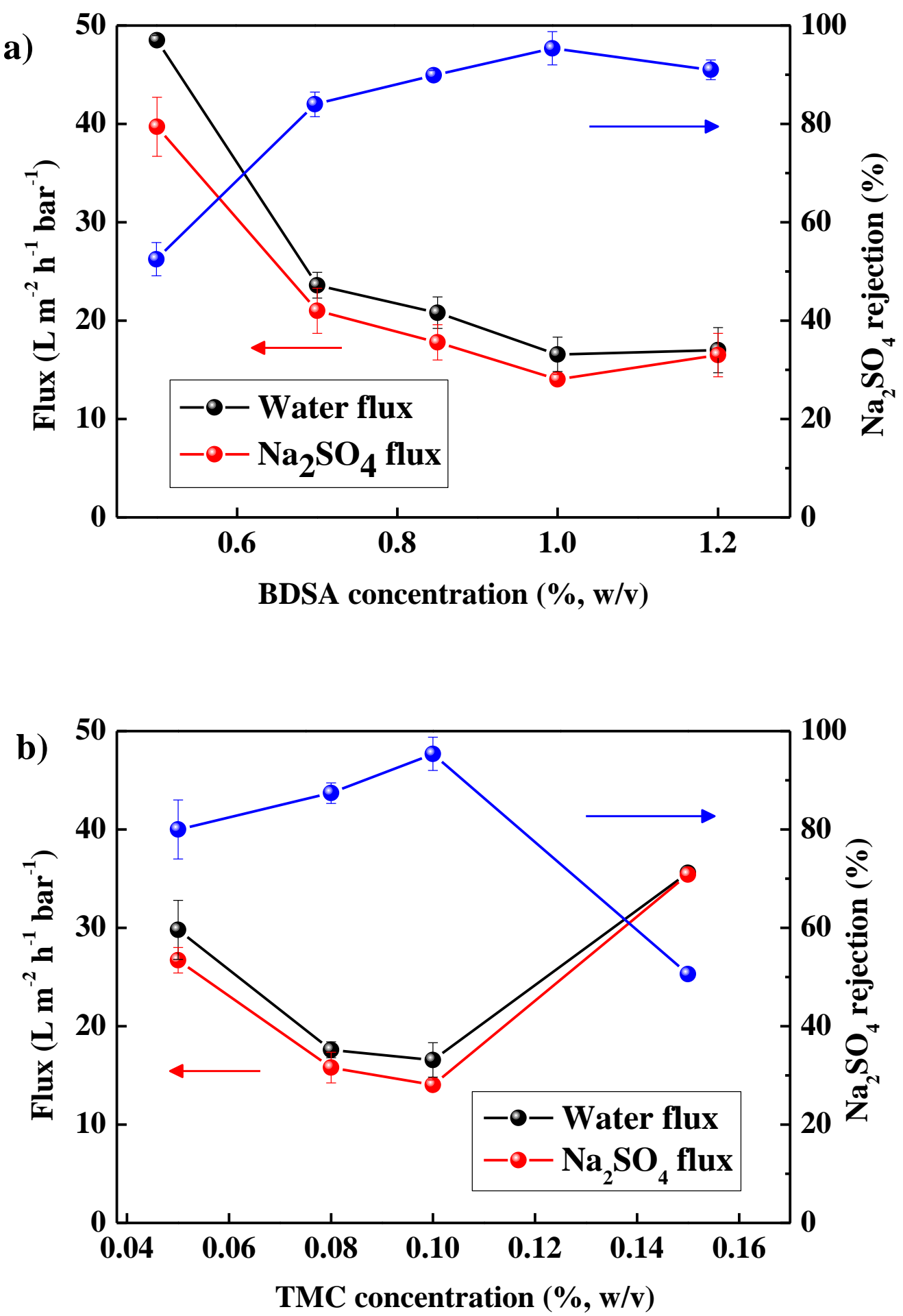

Fig. 1. Effects on the separation performance of TFC membranes of (a) BDSA concentration $\left(\mathrm{C}_{\mathrm{TMC}}\right.$ in $n$-hexane was fixed at $\left.0.1 \%(\mathrm{w} / \mathrm{v})\right)(\mathrm{b}) \mathrm{TMC}$ concentration $\left(\mathrm{C}_{\mathrm{BDSA}}\right.$ in water was fixed at $1.0 \%(\mathrm{w} / \mathrm{v}))$. 
Having evaluated the various feed conditions, TFC-1.0 was selected as the optimized membrane sample for further characterizations. The pure water flux was initially measured as a function of feed pressure, as shown in Fig. 2. The water permeability of TFC-1.0 was found to increase with increasing applied pressures due to enhancement of the driving force for permeation of water. By calculating the inverse value of the slope of the fitting line in Fig. 2, the intrinsic resistance of the membrane $\left(R_{\mathrm{m}}\right)$ was obtained. This value was found to be as low as $2.37 \times 10^{13} \mathrm{~m}^{-1}$ for TFC-1.0, which is an order of magnitude lower than some other reported membranes [41]. This low value indicates weak resistance against hydraulic pressure and as a result, the membranes would show greatly enhanced water permeability under applied pressure.

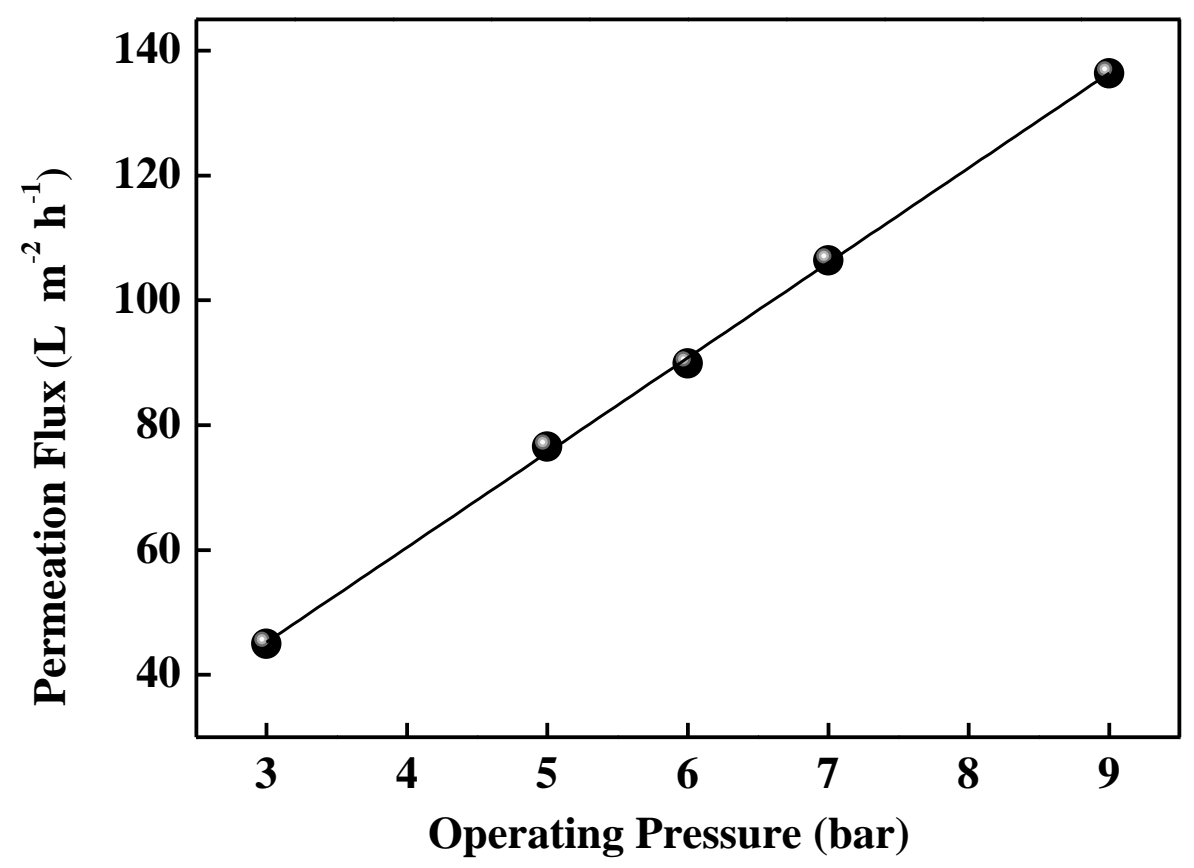

Fig. 2. Water flux of TFC-1.0 under various operating pressures.

A summary of the separation performance of TFC-1.0 with regard to inorganic salts and two organic dyes is shown in Fig. 3. The salt rejection ability follows the order of $\mathrm{Na}_{2} \mathrm{SO}_{4}>\mathrm{NaCl}>\mathrm{MgCl}_{2}>\mathrm{CuSO}_{4}>\mathrm{MgSO}_{4}$, as expected from a typical negatively charged membrane surface. It is well known that the rejection ability in a 
NF process is decided not only by steric hindrance but also by the Donnan effect and dielectric effects [9]. Compared to the higher rejection of $\mathrm{Na}_{2} \mathrm{SO}_{4}$, the relatively lower rejection of $\mathrm{MgCl}_{2}, \mathrm{MgSO}_{4}$ and $\mathrm{CuSO}_{4}$ is mainly due to adsorption of divalent cations. These adsorbed cations help "shield" the negative charges on the membrane surface, resulting in a decrease in the absolute surface potential and a much weakened Donnan effect $[42,43]$. Moreover, the TFC-1.0 membrane also exhibited superior retention performance for dye solutions; the retention for Congo Red and Methylene Blue (their structure showed in Fig. S1.) were as high as $99.0 \%$ and $90.6 \%$, respectively.

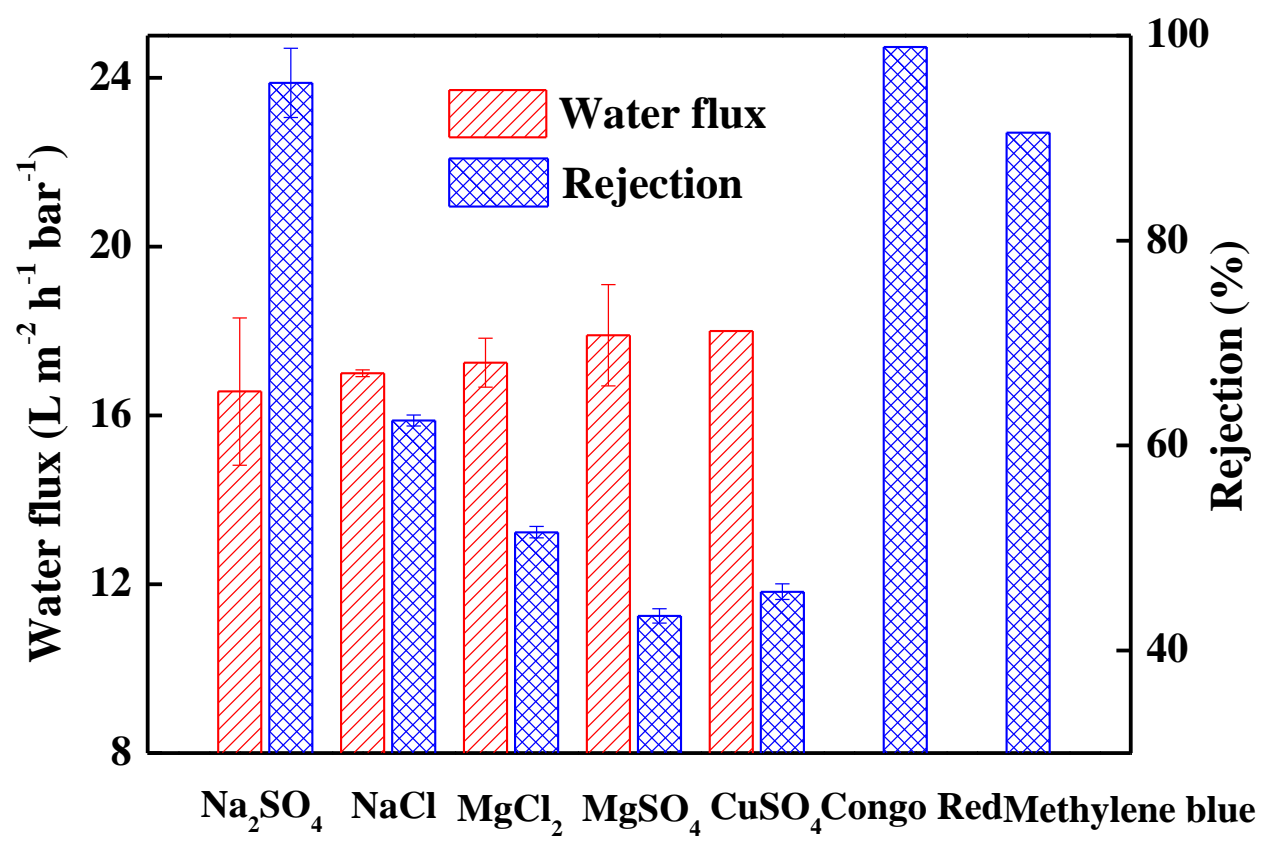

Fig. 3. Separation performance of the TFC-1.0 membrane for different salts and dyes. Test conditions: inorganic salt and dye concentration $=1000 \mathrm{mg} \mathrm{L}^{-1}, 25^{\circ} \mathrm{C}, 0.6 \mathrm{MPa}$.

Table 3 lists the molecular weights and the Stokes radii of the four selected solutes. A linear relationship was obtained between solute rejections and solute Stokes radii, with a high correlation coefficient of 0.94, as shown in Fig. 4 (a). Based on Equation (3), the TFC-1.0 membrane was calculated to have a broad pore size distribution with nearly all the pore radii falling within the range of 0.1 to $0.4 \mathrm{~nm}$, 
consistent with the defined ranges for NF membranes.

Table 3 Molecular weight, the Stokes radii data and Solute rejection of four solutes.

\begin{tabular}{ccccc}
\hline Solute & $\begin{array}{c}\text { Ethylene } \\
\text { glycol }\end{array}$ & $\begin{array}{c}\text { Diethylene } \\
\text { glycol }\end{array}$ & $\begin{array}{c}\text { Triethylene } \\
\text { glycol }\end{array}$ & Glucose \\
\hline Stokes radii, $r_{\mathrm{s}}(\mathrm{nm})$ & 0.235 & 0.291 & 0.334 & 0.365 \\
Molecular weight $(\mathrm{Da})$ & 62.07 & 106.12 & 150.17 & 180.16 \\
Solute rejection, $R_{\mathrm{r}}(\%)$ & 39.73 & 59.84 & 68.00 & 88.12 \\
\hline
\end{tabular}

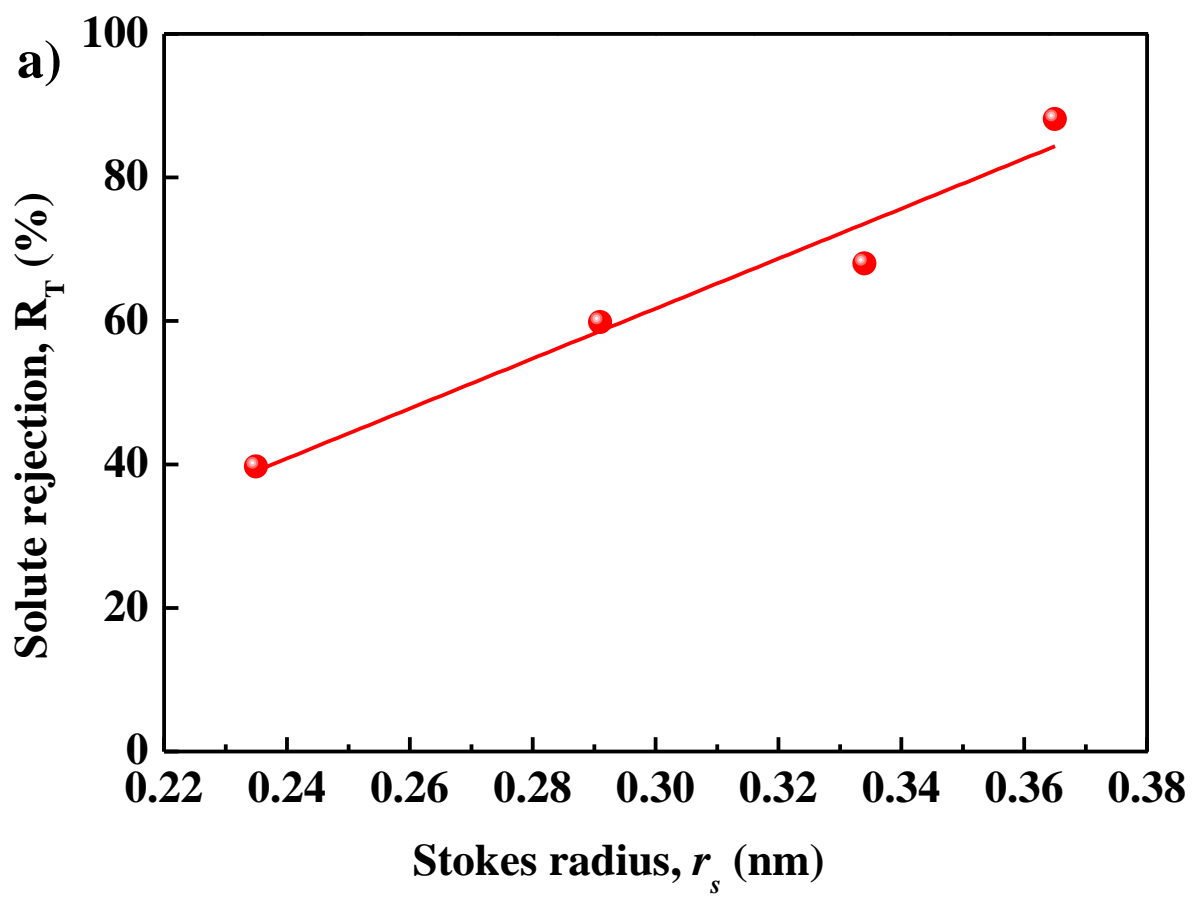




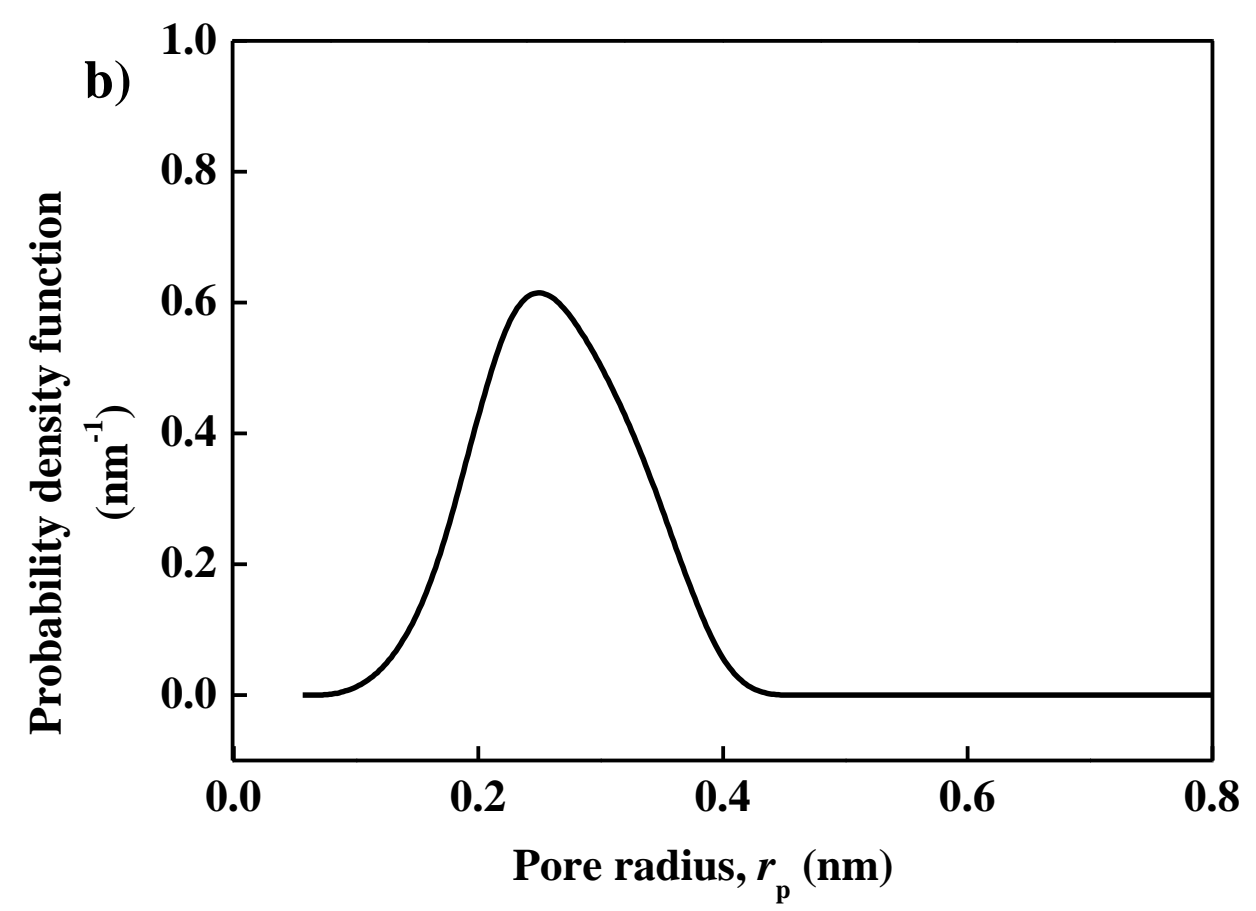

Fig. 4. (a) Effective rejection curves (solute rejection vs. Stokes radii); (b) probability density function curves of TFC-1.0 membrane.

\subsection{Surface characteristics}

\subsubsection{Morphology}

The morphology of the membrane surface considerably affects the membrane separation performance. Fig. 5 and Fig. 6 show the surface morphologies of the TFCs measured by FESEM and AFM. Compared with the virgin PSf substrate (Fig. 5 (a)), no clear pores could be identified in Fig. 5 (b), indicating that the entire TFC membrane surface was successfully coated with the nascent layers. In addition, there were no nodules or projections observed in all the surface images of TFC membranes, indicating their smooth nature.

Similar results were also obtained by AFM, where the average plane roughness (Ra) was found to be within a narrow range of 1.51 to $2.72 \mathrm{~nm}$. While it is known that a rougher membrane surface results in higher permeation flux of NF or RO membranes due to the increased surface area $[44,45]$, in this study, the high water flux should be attributed to the strong hydrophilicity of the sulfonic acid groups. 
Furthermore, as shown in Fig. 5 (d), the upper barrier layer of the TFC-1.0 membrane was roughly estimated to be $150 \mathrm{~nm}$ in thickness, compared with the PSf substrate in Fig. 5 (c).

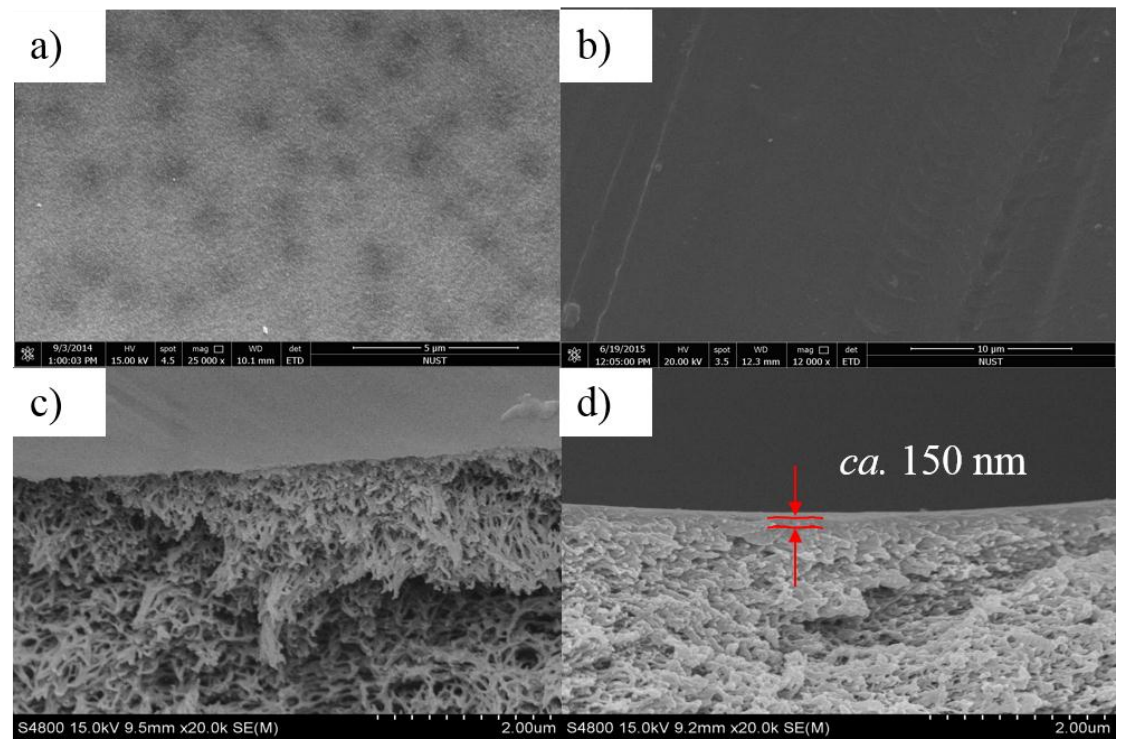

Fig. 5. FESEM images of: top surface of (a) PSf membrane; (b) TFC-1.0 membrane, cross-section of (c) PSf membrane; (d) TFC-1.0 membrane. 

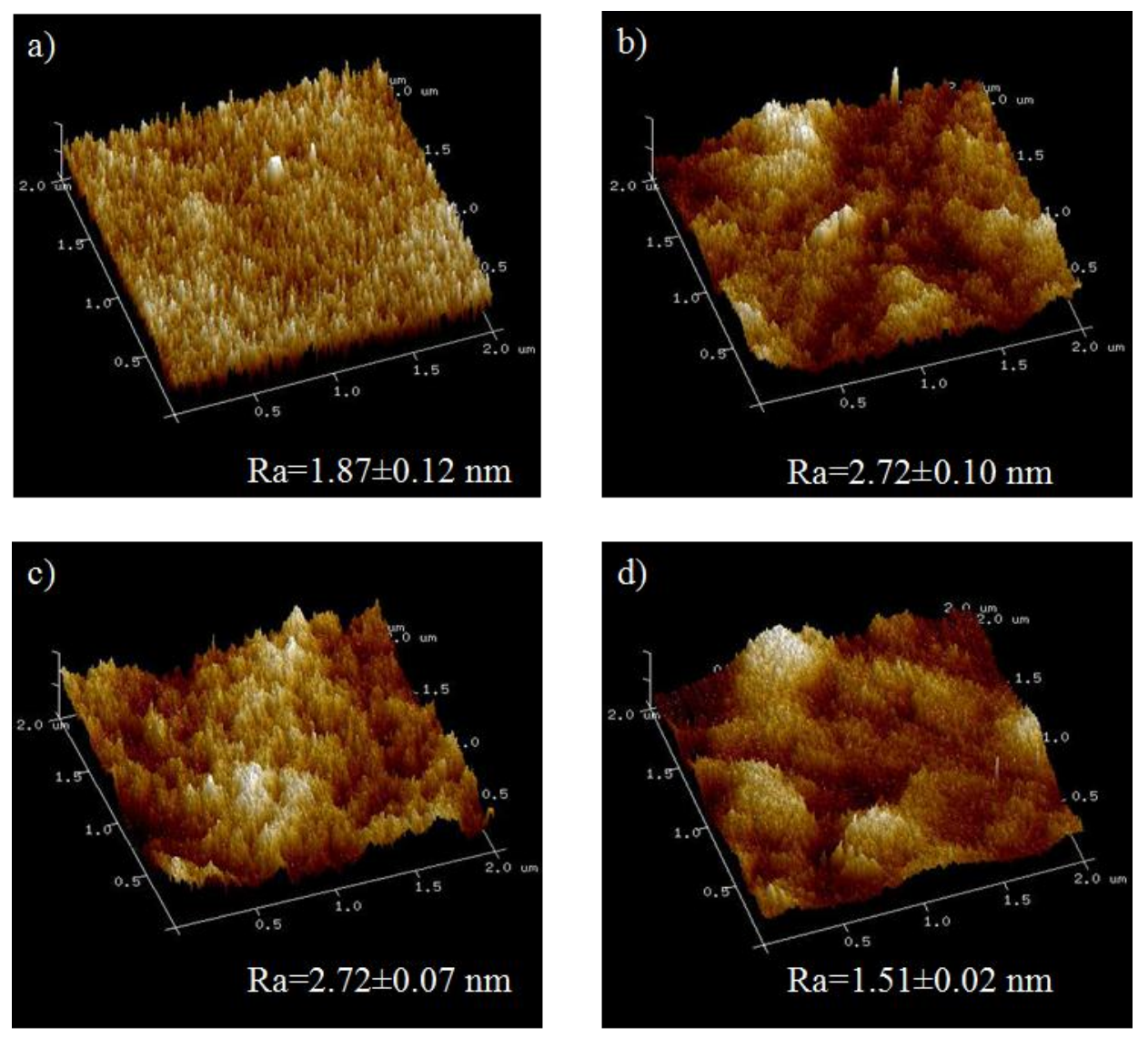

Fig. 6. AFM morphology of (a) PSf, (b) TFC-0.5, (c) TFC-1.0 and (d) TFC-1.2 membranes.

\subsubsection{Contact Angle and Zeta Potential}

Water contact angle (CA) is closely related to hydrophilic characteristics of the TFC surface, and a low CA generally indicates high hydrophilicity. Taking the polar nature of the sulfonic acid groups into account, the hydrophilicity of the membrane surface is expected to be greatly enhanced. However, the experimental results were quite contrary to our expectations, i.e., the CA values progressively increased with the increase in BDSA concentration (Fig. 7), indicating higher hydrophobicity. The highest CA value was found to $86.5 \pm 1.9$ degrees for TFC-1.0, which was quite different from the expected value. To explain this unusual behavior, we proposed that most of the "- $\mathrm{SO}_{3} \mathrm{H}$ " groups are enclosed by the hydrophobic polymer backbones. To test this assumption, the Zeta potential of the TFC membrane was firstly investigated, as shown in Fig. 8. The TFC membrane surface showed amphiprotic behaviour over 
the entire $\mathrm{pH}$ range with the low isoelectric point at $\mathrm{pH}$ 3.0. The surface was found to be positively charged below $\mathrm{pH} 3.0$ due to protonation of the amine groups. Interestingly, it was observed that the TFC membrane exhibited greater potential values $(-15.65$ to $-17.48 \mathrm{mV}$ with $\mathrm{pH}$ 6.0-7.6) than the virgin substrate (-20.36 to -23.99 $\mathrm{mV}$ with $\mathrm{pH}$ 6.0-7.5), which indicates that the membrane has lower surface charge. Considering the $\mathrm{pKa}$ value of benzenesulfonic acid $(0.70,[46])$, the introduction of "- $\mathrm{SO}_{3} \mathrm{H}$ " groups should effectively increase the surface negative charge, since the hydrogens completely dissociate under neutral condition. The most likely explanation for this anomaly is that the amount of the sulfonic acid groups on the outer most surface are rather limited. Also, some of the carboxyl groups are likely "blocked" by the polymer matrix due to the chain rigidity. The observed Zeta potential results correlate well with our aforementioned assumption.

Anti-fouling property provides another effective approach towards evaluating the membrane hydrophilicity since the organic pollutants tend to be absorbed on hydrophobic surfaces. The water flux decreased to $85 \%$ of the original level after a continuous filtration test for 48 hours as shown in Fig. S3. Also, after an extreme fouling test with bovine serum albumin (BSA) solution, the corresponding total flux decline ratio $\left(D R_{\mathrm{t}}\right)$ reached to the relatively high level of $37.7 \%$, indicating its vulnerable nature. Nevertheless, the flux recovery ratio $(F R R)$ was still maintained at an acceptably high level of $76.0 \%$, after a back-washing process (Fig. S4). 


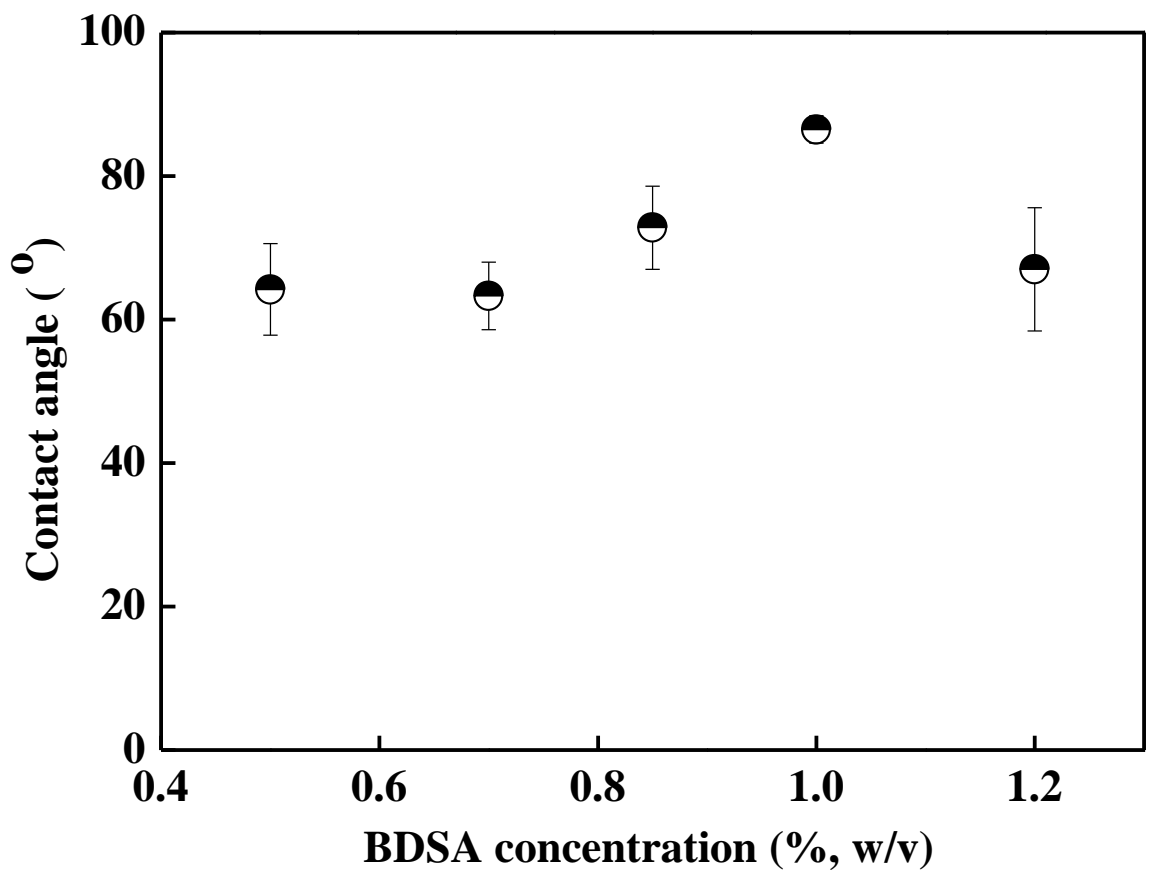

Fig. 7. Water contact angle of TFCs with different BDSA concentration.

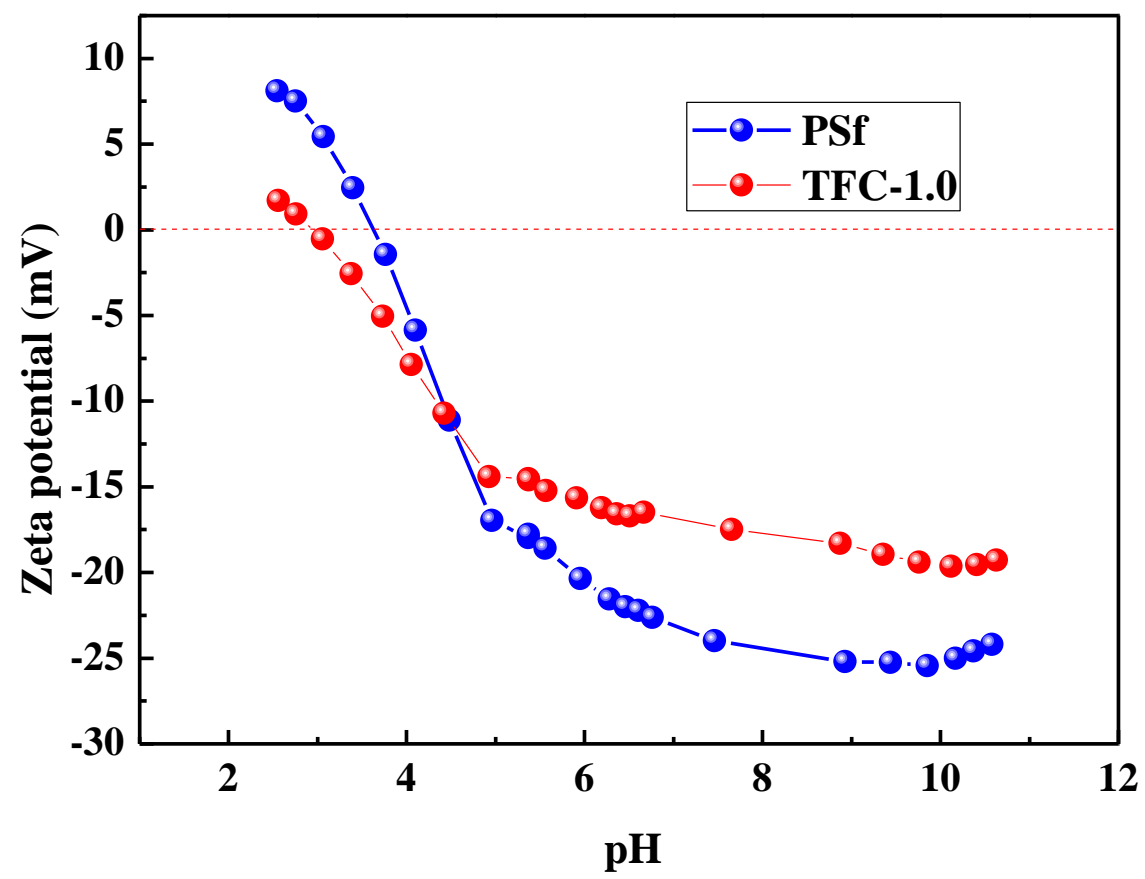

Fig. 8. Zeta potential of TFC-1.0 membrane at various $\mathrm{pH}$ values.

\subsection{DPD discussion}

Based on the above results and the assumption that the sulfonic acid groups are not located in the outer face, a more detailed study was performed using a mesoscopic 
simulation method. For the construction and effectiveness of the DPD simulations, the repulsion between the beads is of great importance, since it directly determines the compatibility of the different moieties. As mentioned previously, the repulsion parameters were calculated using the Flory-Huggins parameters, and the obtained results are presented in Table S1. The binary repulsion parameter is the maximum repulsion between two particles $i$ and $j$, and the closer to the self-repulsion value $\left(a_{\mathrm{ii}}=\right.$ 25), the less repulsion particles have towards each other. In most cases, DPD methods can only be used to obtain a qualitative description rather than a quantitative analysis, since the chemical bond formation could not be depicted. Therefore, it is essential to determine the repulsion, particularly between the beads A (phenyl amine group) and C (benzoyl chloride group). We were happy to note that the calculated $a_{\mathrm{ij}}$ value for these two beads was 25.46, indicating the low repulsion and excellent miscibility of the two functional groups. In other words, once the two beads are connected to each other, it could be considered as an amide bond formation although it is not a "true state" during a mesoscopic simulation.

The initial configuration of the DPD dynamics is presented in Fig. 9 (a). As it can be seen, all the BDSA and TMC monomers are well dispersed throughout the water and oil phases, respectively. After a 400,000 steps (20,000 ps) simulation period, the lowest energy frame was obtained, where all of the BDSA monomers moved to the oil-water interface. In contrast, the TMC monomers still maintained their disorderliness in the organic phase, as shown in Fig. 9 (b). It is generally accepted that the monomers in the aqueous solutions always diffuse to the organic phase during the IP process. Once the solubility reaches a certain point, the polymer would precipitate at the interface on the porous substrate $[15,47]$. With this assumption, the coarse grain mapping and the model construction proposed here are quite adequate, since all polymers were generated at the middle region of the simulation box. 

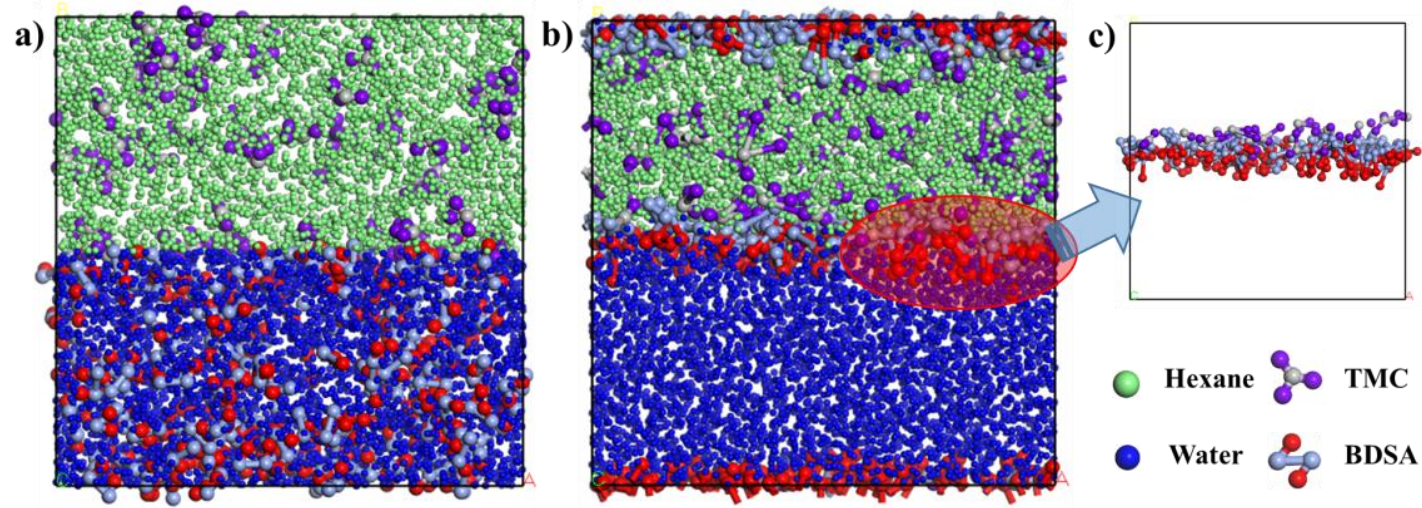

Fig. 9. Snapshots of DPD simulations of oil-water interface a) before and b) after the IP process; c) an enlarged snapshot of the interface after IP process, where the excessive hexane, water and monomeric beads have been removed for clarity.

Additionally, it can be observed that the polymer generated at the interface has most of the sulfonic acid groups facing down towards the water phase, whereas the benzene rings together with a few "-COOH" groups (hydrolyzed from "-COCl") serve as a cover to the polymer matrix (Fig. 9 (c)). It is expected that the BDSA monomers diffuse to the top of the water solution, with partial affinity (due to aniline moieties) towards the oil phase and partial affinity for the water (due to sulfonic acid groups). However, the phenyl backbone of the BDSA has a relatively strong affinity for the oil phase, and thus the "ph-CONH-ph" polymeric units immediately form at the interface while most of the pendant "- $\mathrm{SO}_{3} \mathrm{H}$ " groups remain in the water, as revealed by the concentration profiles in Fig. 10. Taking the structural configuration into account, i.e., the full phenyl-phenyl bond of the BDSA monomer indicating the chain rigidity, there is likely some $\pi-\pi$ stacking interactions between the aromatic moieties (the light blue bead aggregated at the top of the polymer layer), which tend to produce sufficient pores inside the newly formed polymers. The BDSA monomers continued diffusing though these pores to react with TMC and eventually the sulfonated polyamide layer was obtained. Notably, in most cases, the "- $\mathrm{SO}_{3} \mathrm{H}$ " groups were enclosed within the polymer skeletons. 


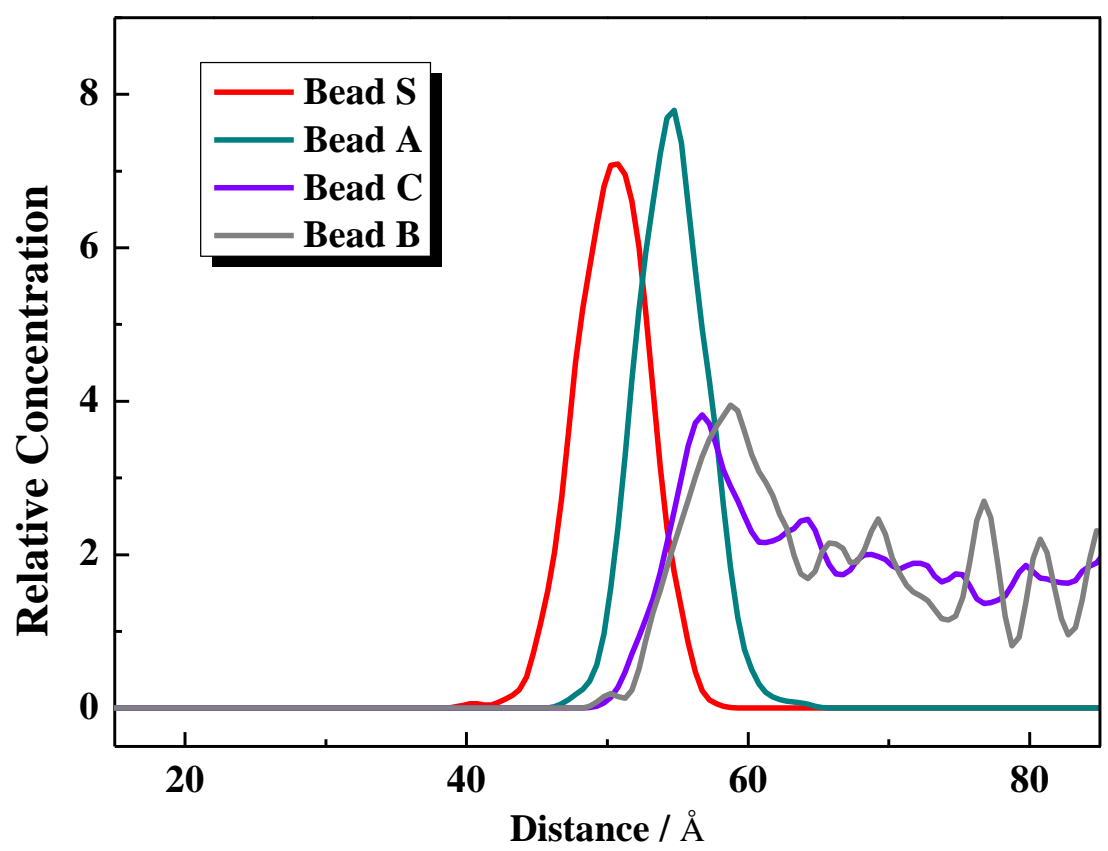

Fig. 10. Concentration profile of the four beads, S, A, C and B in the plane $\left(\begin{array}{lll}0 & 1 & 0\end{array}\right)$ in the simulation box $(100 \times 100 \times 100) \AA^{3}$.

Our findings in the simulation studies strongly support the results of contact angle and Zeta potential. Therefore, we propose a new schematic model of our membrane surface structure in this work (Fig. 11). As shown in Fig. 11, the proposed membrane structure has the sulfonic polyamide (SPA) film consisting of protuberances and ridges, which form a relatively smooth dense layer with several tunnels inside them. Most of the sulfonic acid groups serve as the polymer brushes, and are located at the inner face of the barrier layer to generate several small "negative charge aggregated" cavities. In contrast, the lower region, i.e., the region where the polymer is in contact with PSf substrate, is not a continuous layer and has many pores on it, which enable the passage of the excess BDSA monomers during the IP process. Due to the strong hydrophilicity of the sulfonic acid groups, water molecules easily move from the bulk of the layer to the outer surface, enter the cavities through the pores on the dense layer, and form a thin water layer attached to the inner edge. Notably, this layer provides a hydraulic drag to the solute in addition to the electrostatic repulsive force, thus, the multivalent ions are rejected once they reach the cavities. Eventually, the progressively higher ionic strength could cause an inverse concentration gradient, 
which indicates a slight internal concentration polarization (ICP) effect, as revealed above in Fig. 1. Overall, on the basis of the above discussions, it is clear that our SPA membranes exhibit some hydrophobicity and have lower surface charge compared to the parent PSf support under neutral conditions. Until now, only the external surface properties of these membranes have been characterized and studied, and further research in our laboratory is in progress to obtain a more in-depth understanding of the membrane structure. Nonetheless, the SPA membranes display very effective filtration performance due to the introduction of "- $\mathrm{SO}_{3} \mathrm{H}$ " groups.

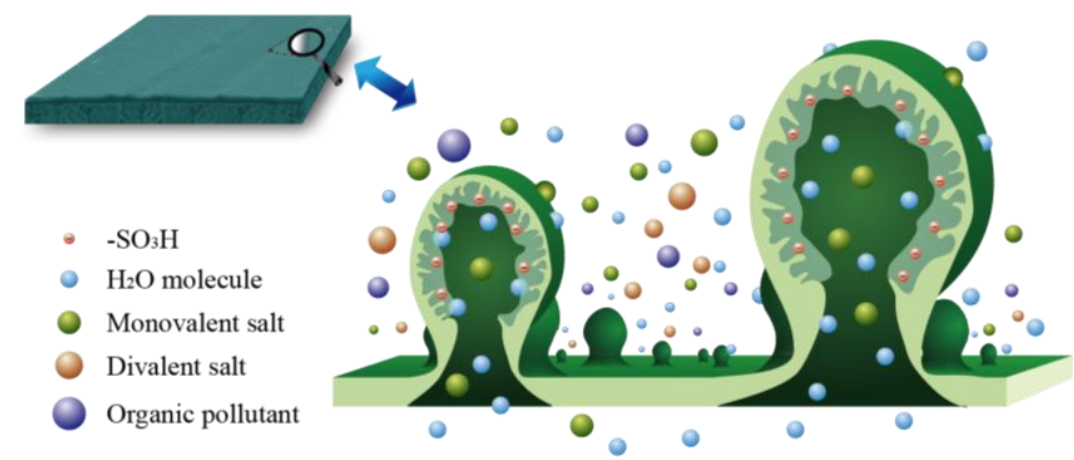

Fig. 11. A new schematic model of TFC membrane surface structure via BDSA and TMC.

\section{Conclusion}

In this study, a series of TFC membranes were prepared by interfacial polymerization of BDSA with TMC on polysulfone ultrafiltration supports. By adjusting the BDSA and TMC concentrations, the optimized membrane (TFC-1.0) was obtained, whose $\mathrm{Na}_{2} \mathrm{SO}_{4}$ rejection ability reached up to $95.4 \%$ with a high flux of 16.6 $\mathrm{L} \mathrm{m}^{-2} \mathrm{~h}^{-1} \mathrm{bar}^{-1}$. Rejection properties of the NF membranes towards various salts and dye solutes were assessed, and it was found that the salt retention ability follows the order of $\mathrm{Na}_{2} \mathrm{SO}_{4}>\mathrm{NaCl}>\mathrm{MgCl}_{2}>\mathrm{CuSO}_{4}>\mathrm{MgSO}_{4}$. Pore size (0.1-0.4 nm) of the TFC membranes was also evaluated separately, and the value was found to be within the acceptable range for NF membranes. XPS analysis successfully confirmed the highest crosslinking degree of TFC-1.0 from the chemical composition. FESEM, AFM and water contact angle measurements confirmed that the TFC membranes possessed smooth and hydrophobic surfaces. Furthermore, DPD simulation was 
performed to obtain the monomer configuration with regard to the IP process. The simulation showed that most of the "- $\mathrm{SO}_{3} \mathrm{H}$ " groups were facing down towards the aqueous solution, and covered by the rigid polymer backbones. Therefore, we proposed a new schematic model of our membrane surface structure in this study, in which most of the sulfonic acid groups are located at the inner face of the protuberances to generate several small "negative charge aggregated" particles. The water molecules are easily able to enter the polymer matrix due to the strong hydrophilicity of sulfonic acid groups, whereas the salts and organics are rejected due to the electrostatic repulsion. Overall, the results of this study indicate that the sulfonated TFC membranes are highly promising candidates for future NF applications.

\section{Acknowledgment}

This work was financially supported by the NSFC (21406117), the Natural Science Foundation of Jiangsu Province (BK20140782), National Science Foundation for Post-doctoral Scientists of China (2014M561652), the Jiangsu Planned Projects for Postdoctoral Research Funds (1401045B), Scientific Research Foundation for Returned Scholars (Ministry of Education of China), PAPD and the Fundamental Research Funds for the Central Universities (30920140122008, 30915011306). The

author also would like to thank Prof. Q. F. An in Zhejiang University for Zeta Potential measurement. 


\section{Nomenclature}

$\begin{array}{ll}\text { NF } & \text { nanofiltration } \\ \text { SPA } & \text { sulfonic polyamide } \\ \text { TFC } & \text { thin-film-nanocomposite } \\ \text { IP } & \text { interfacial polymerization } \\ \text { DFT } & \text { density functional theory } \\ \text { MD } & \text { molecular dynamics } \\ \text { BDSA } & \text { 2,2'-benzidinedisulfonic acid } \\ \text { TMC } & \text { trimesoyl chloride } \\ \text { DPD } & \text { dissipative particle dynamics } \\ \text { PSf } & \text { polysulfone } \\ J & \text { water flux } \\ \text { Ra } & \text { average plane roughness } \\ \zeta & \text { surface zeta potential } \\ R & \text { salts rejection } \\ \chi_{\mathrm{ij}} & \text { cohesive energy density } \\ a_{\mathrm{ij}} & \text { internal concentration polarization } \\ \text { ICP } & \text { the intrinsic resistance of membrane } \\ R_{\mathrm{m}} & \text { total flux decline ratio } \\ D R & \text { flux recovery ratio } \\ & \end{array}$




\section{References}

[1] M. Elimelech, W. A. Phillip, The future of seawater desalination: energy, technology, and the environment, Science 333 (2011) 712-717.

[2] X. Qu, P. J. J. Alvarez, Q. Li, Applications of nanotechnology in water and waste water treatment, Water Res. 47 (2013) 3931-3946.

[3] A. W. Mohammad, Y. H. Teow, W. L. Ang, Y. T. Chung, D. L. Oatley-Radcliffe, N. Hilal, Nanofiltration membranes review: Recent advances and future prospects, Desalination 369 (2015) 226-254.

[4] W. J. Lau, A. F. Ismail, N. Misdan, M. A. Kassim, A recent progress in thin film composite membrane: A review, Desalination 287 (2012) 190-199.

[5] M. M. Pendergasta, E. M. V. Hoek, A review of water treatment membrane nanotechnologies, Energy Environ. Sci. 4 (2011) 1946-1971.

[6] G. M. Geise, H. S. Lee, D. J. Miller, B. D. Freeman, J. E. McGrath, D. R. Paul, Water purification by membranes: The role of polymer science, J. Polym. Sci., Part B: Polym. Phys. 48 (2010) 1685-1718.

[7] B. V. Bruggen, M. Mänttäri, M. Nyström, Drawbacks of applying nanofiltration and how to avoid them: A review, Sep. Purif. Technol. 63 (2008) 251-263.

[8] K. P. Lee, T. C. Arnot, D. Mattia, A review of reverse osmosis membrane materials for desalination - Development to date and future potential, J. Membr. Sci. 370 (2011) 1-22.

[9] Y. Lv, H. C. Yang, H. Q. Liang, L. S. Wan, Z. K. Xu, Nanofiltration membranes via co-deposition of polydopamine/polyethylenimine followed by cross-linking, J. Membr. Sci. 476 (2015) 50-58.

[10] W .P. Zhu, J. Gao, S. P. Sun, S. Zhang, T. S. Chung, Poly(amidoamine) dendrimer (PAMAM) grafted on thin film composite (TFC) nanofiltration (NF) hollow fiber membranes for heavy metal removal, J. Membr. Sci. 438 (2015) 117-126.

[11] Y. F. Li, Y. L. Su, Y. N. Dong, X. T. Zhao, X. He, R. N. Zhang, J. J. Zhao, X. C. Fan, Z.Y. Jiang, Antifouling, High-Flux Nanofiltration Membranes Enabled by Dual Functional Polydopamine, ACS Appl. Mater. Interfaces 6 (2014) 5548-5557.

[12] A. M. Saenz de Jubera, J. H. Herbison, Y. Komaki, M. J. Plewa, J. S. Moore, D.G. Cahill, B. J. Mariñas, Development and Performance Characterization of a Polyamide Nanofiltration 
Membrane Modified with Covalently Bonded Aramide Dendrimers, Environ. Sci. Technol. 47 (2013) 8642-8649.

[13] T. Y. Wang, Y. Q. Yang, J. F. Zheng, Q. F. Zhang, S. B. Zhang, A novel highly permeable positively charged nanofiltration membrane based on a nanoporous hyper-crosslinked polyamide barrier layer, J. Membr. Sci. 448 (2013) 180-189.

[14] Y. F. Mi, Q. Zhao, Y. L. Ji, Q. F. An, C. J. Gao. A novel route for surface zwitterionic functionalization of polyamide nanofiltration membranes with improved performance, J. Membr. Sci. 490 (2015) 311-320.

[15] A. G. Fane, R. Wang, M. X. Hu, Synthetic Membranes for Water Purification: Status and Future, Angew. Chem. 54 (2015) 3368-3386.

[16] Q. F. An, W. D. Sun, Q. Zhao, Y.L. Ji, C.J. Gao, Study on a novel nanofiltration membrane prepared by interfacial polymerization with zwitterionic amine monomers, J. Membr. Sci. 431 (2013) 171-179.

[17] T. Y. Liu, Z. H. Liu, R. X. Zhang, Y. Wang, B. V. Bruggen, X. L. Wang, Fabrication of a thin film nanocomposite hollow fiber nanofiltration membrane for wastewater treatment, J. Membr. Sci. 488 (2015) 92-102.

[18] H. Yan, X. P. Miao, J. Xu, G. Y. Pan, Y. Zhang, Y. T. Shi, M. Guo, Y. Q. Liu, The porous structure of the fully-aromatic polyamide film in reverse osmosis membranes, J. Membr. Sci. 475 (2015) 504-510.

[19] J. Zuo, J. Y. Lai, T. S. Chung, In-situ synthesis and cross-linking of polyamide thin film composite (TFC) membranes for bioethanol applications, J. Membr. Sci. 458 (2014) 47-517.

[20] Y. F. Li, Y. L. Su, X. T. Zhao, R. N. Zhang, J. J. Zhao, X. C. Fan, Z. Y. Jiang, Surface fluorination of polyamide nanofiltration membrane for enhanced antifouling property, J. Membr. Sci. 455 (2014) 15-23.

[21] X. Z. Wei, X. Kong, J. Yang, G. L. Zhang, J. Y. Chen, J. Wang, Structure influence of hyperbranched polyester on structure and properties of synthesized nanofiltration membranes, J. Membr. Sci. 440 (2013) 67-76.

[22] J. X. Qin, S. S. Lin, S. Q. Song, L. Zhang, H. L. Chen, 4-Dimethylaminopyridine Promoted Interfacial Polymerization between Hyperbranched Polyesteramide and Trimesoyl Chloride for Preparing Ultralow-Pressure Reverse Osmosis Composite Membrane, ACS Appl. Mater. 
Interfaces 5 (2013) 6649-6656.

[23] Y. F. Li, Y. L. Su, Y. N. Dong, X. T. Zhao, Z. Y. Jiang, R. N. Zhang, J. J. Zhao, Separation performance of thin-film composite nanofiltration membrane through interfacial polymerization using different amine monomers, Desalination 333 (2014) 59-65.

[24] W. X. Fang, L. Shi, R. Wang, Interfacially polymerized composite nanofiltration hollow fiber membranes for low-pressure water softening, J. Membr. Sci. 430 (2013) 129-139.

[25] H. Ebro, Y. M. Kim, J. H. Kim, Molecular dynamics simulations in membrane-based water treatment processes: A systematic overview, J. Membr. Sci. 438 (2013) 112-125.

[26] Z. E. Hughes, J. D. Gale, Molecular dynamics simulations of the interactions of potential foulant molecules and a reverse osmosis membrane, J. Mater. Chem. 22 (2012) 175-184.

[27] R. Lo, A. Bhattacharya, B. Ganguly, Probing the selective salt rejection behavior of thin film composite membranes: A DFT study, J. Membr. Sci. 436 (2013) 90-96.

[28] Y. Xiang, Y. L. Liu, B. X. Mi, Y. S. Leng, Hydrated Polyamide Membrane and Its Interaction with Alginate: A Molecular Dynamics Study, Langmuir 29 (2013) 11600-11608.

[29] E. Harder, D. E. Walters, Y. D. Bodnar, R. S. Faibish, B. Roux, Molecular Dynamics Study of a Polymeric Reverse Osmosis Membrane, J. Phys. Chem. B 113 (2009) 10177-10182.

[30] Z. W. Lv, J. H. Hu, X. Zhang, L. J. Wang, Enhanced surface hydrophilicity of thin-film composite membranes for nanofiltration: an experimental and DFT study, Phys. Chem. Chem. Phys. 17 (2015) 24201-24209.

[31] J. Gao, S. P. Sun, W. P. Zhu, T. S. Chung, Polyethyleneimine (PEI) cross-linked P84 nanofiltration (NF) hollow fiber membranes for $\mathrm{Pb}^{2+}$ removal, J. Membr. Sci. 452 (2014) 300-310.

[32] J. Su, Q. Yang, J. F. Teo, T. S. Chung, Cellulose acetate nanofiltration hollow fiber membranes for forward osmosis processes, J. Membr. Sci. 355 (2010) 36-44.

[33] S. P. Sun, T. A. Hatton, T. S. Chung, Hyperbranched polyethyleneimine induced cross-linking of polyamide-imide nanofiltration hollow fiber membranes for effective removal of ciprofloxacin, Environ. Sci. Technol. 45 (2011) 4003-4009.

[34] D. R. Gutiérrez, C. N. Draghi, N. Pannacci, L. V. Castro, F. Á. Ramírez, B. Creton, Surface Photografting of Acrylic Acid on Poly(dimethylsiloxane). Experimental and Dissipative Particle Dynamics Studies. Langmuir 31 (2015) 1400-1409.

[35] C. M. Hansen, Hansen Solubility Parameters A User's Handbook, second ed., Taylor and 
Francis, Boca Raton, 2007.

[36] L. L. Shao, Q. F. An, Y. L. Ji, Q. Zhao, X. S. Wang, B. K. Zhu, C. J. Gao, Preparation and characterization of sulfated carboxymethyl cellulose nanofiltration membranes with improved water permeability, Desalination 338 (2014) 74-83.

[37] J. J. Zhao, Y. L. Su, X. He, X. T. Zhao, Y. F. Li, R. N. Zhang, Z. Y. Jiang, Dopamine composite nanofiltration membranes prepared by self-polymerization and interfacial polymerization, J. Membr. Sci. 465 (2014) 41-48.

[38] C. C. Feng, J. Xu, M. M. Li, Y. Y. Tang, C. J. Gao, Studies on a novel nanofiltration membrane prepared by cross-linking of polyethyleneimine on polyacrylonitrile substrate, $\mathrm{J}$. Membr. Sci. 451 (2014) 103-110.

[39] R. Ding, H. Q. Zhang, Y. F. Li, J.T. Wang, , B. B. Shi, H. Mao, J. C. Dang, J. D. Liu, Graphene oxide-embedded nanocomposite membrane for solvent resistant nanofiltration with enhanced rejection ability, Chem. Eng. Sci. 138 (2015) 227-238.

[40] H. Mao, H. Q. Zhang, Y. F. Li, Y. B. Xue, F. Pei, J. T. Wang, J. D. Liu, Tunable Solvent Permeation Properties of Thin Film Nanocomposite Membrane by Constructing Dual-Pathways Using Cyclodextrins for Organic Solvent Nanofiltration, ACS Sustainable Chem. Eng. 3 (2015) 1925-1933.

[41] G. N. B. Baroña, J. Lim, B. Jung, High performance thin film composite polyamide reverse osmosis membrane prepared via m-phenylenediamine and 2,2'-benzidinedisulfonic acid, Desalination 291 (2012) 69-77.

[42] L. M. Jin, S. L. Yu, W. X. Shi, X. S. Yi, N. Sun, Y. L. Ge, C. Ma, Synthesis of a novel composite nanofiltration membrane incorporated $\mathrm{SiO}_{2}$ nanoparticles for oily wastewater desalination, Polymer 53 (2012) 5295-5303.

[43] M. Aiba, T. Tokuyama, S. Baba, H. Matsumoto, H. Tomioka, T. Higashihara, M. Ueda, Improvement in semipermeable membrane performance of wholly aromatic polyamide through an additive processing strategy, J. Polym. Sci., Part A: Polym. Chem. 52 (2014) 1275-1281.

[44] M. Hirose, H. Ito, Y. Kamiyama, Effect of skin layer surface structures on the flux behaviour of RO membranes, J. Membr. Sci. 121 (1996) 209-109.

[45] V. Freger, Nanoscale Heterogeneity of Polyamide Membranes Formed by Interfacial Polymerization, Langmuir 19 (2003) 4791-4797. 
[46] D. R. Lide, Handbook of Chemistry and Physics, 84th ed., Taylor and Francis, Boca Raton, 2004.

[47] P. W. Morgan, S. L. Kwolek, Interfacial polycondensation. II. Fundamentals of polymer formation at liquid interfaces, J. Polym. Sci., Part A: Polym. Chem. 34 (1996) 531-559. 


\section{Figure Captions}

Scheme 1 The reaction process of interfacial polymerization between BDSA and TMC.

Fig. 1. Effects on the separation performance of TFC membranes of (a) BDSA concentration $\left(\mathrm{C}_{\mathrm{TMC}}\right.$ in $n$-hexane was fixed at $\left.0.1 \%(\mathrm{w} / \mathrm{v})\right)$ (b) TMC concentration $\left(\mathrm{C}_{\mathrm{BDSA}}\right.$ in water was fixed at $\left.1.0 \%(\mathrm{w} / \mathrm{v})\right)$.

Fig. 2. Water flux of TFC-1.0 under various operating pressures.

Fig. 3. Separation performance of the TFC-1.0 membrane for different salts and dyes. Test conditions: inorganic salt and dye concentration $=1000 \mathrm{mg} \mathrm{L}^{-1}, 25{ }^{\circ} \mathrm{C}, 0.6 \mathrm{MPa}$.

Fig. 4. (a) Effective rejection curves (solute rejection vs. Stokes radii); (b) probability density function curves of TFC-1.0 membrane.

Fig. 5. FESEM images of: top surface of (a) PSf membrane; (b) TFC-1.0 membrane, cross-section of (c) PSf membrane; (d) TFC-1.0 membrane.

Fig. 6. AFM morphology of (a) PSf, (b) TFC-0.5, (c) TFC-1.0 and (d) TFC-1.2 membranes.

Fig. 7. Water contact angle of TFCs with different BDSA concentration.

Fig. 8. Zeta potential of TFC-1.0 membrane at various $\mathrm{pH}$ values.

Fig. 9. Snapshots of DPD simulations of oil-water interface a) before and b) after the IP process; c) an enlarged snapshot of the interface after IP process, where the excessive hexane, water and monomeric beads have been removed for clarity.

Fig. 10. Concentration profile of the four beads, S, A, C and B in the plane $\left(\begin{array}{lll}0 & 1 & 0\end{array}\right)$ in the simulation box $(100 \times 100 \times 100) \AA^{3}$.

Fig. 11. A new schematic model of TFC membrane surface structure via BDSA and TMC. 




A sulfonated polyamide thin-film composite nanofiltration membrane was fabricated with a high water permeability of $16.6 \mathrm{~L} \mathrm{~m}^{-2} \mathrm{~h}^{-1} \mathrm{bar}^{-1}$ and rejection to $\mathrm{Na}_{2} \mathrm{SO}_{4}$ of $95 \%$. 